\title{
La ressource forestière française en chênes rouvre et pédonculé. Analyse et explication historique
}

\author{
F Ningre 1, $R$ Doussot 2 \\ 1 Équipe de recherches sur la croissance et la production, \\ INRA, centre de recherches de Nancy, 54280 Champenoux; \\ 2 SERFOB, 45042 Orléans, France
}

(Reçu le 6 janvier 1993; accepté le 2 juin 1993)

\begin{abstract}
Résumé - Le chêne pédonculé et le chêne sessile couvrent $31 \%$ de la surface boisée de production. Par le passé, pour faire face à la pénurie de bois, le besoin s'est fait sentir de réglementer l'exploitation des forêts, le mode d'exploitation du "tire et aire" entériné par le premier code forestier de 1376, ressemblait à nos aménagements en taillis sous futaie et taillis simple. Les donnée; de l'Inventaire forestier national montrent qu'aujourd'hui encore les taillis et taillis scus futaie sont largement majoritaires (fig 1). Ainsi, l'exemple donné, en forêt domaniale, de la conversion en futaie régulière, souvent mise à mal puisque dans leur majorité les futaies ont moins de 120 ans (fig 4), n'a pas eu les effets escomptés sur les principaux propriétaires de la chênaie que sont les privés et les collectivités publiques (fig 2). L'excellente qualité technologique des grumes de taillis sous futaie (fig 6) est un des éléments explicatifs du maintien de ce régime.
\end{abstract}

chêne / histoire / sylviculture / ressource / qualité du bois

Summary - The French forest resource of sessile and pedunculate oak; an analysis in the light of past history. Pedunculate oak and sessile oak cover $31 \%$ of the forested area used for yield. In the past, because of wood shortage, it was necessary to regulate forestry harvesting; the "tire and aire" forestry harvesting was ratified by the first forestry code of 1376 and was similar to the present management as coppice with standards and simple coppice systems. The IFN (National Forestry Inventory) data show that nowadays both coppices and coppices with standards are largely dominant (fig 1). Hence the example given of a state forest its conversion into an even-aged forest, is often difficult since the majority of high forests are $<120 \mathrm{yr}$ old (fig 4) and has the expected effects on the main oak-wood owners: ie private owners and local communities. The excellent technological quality of the tree trunks from coppice with standards (fig 6) is one reason why the use of such a system has been maintained.

oak / history / silviculture / resource / wood quality 


\section{INTRODUCTION}

Le chêne, feuillu précieux par excellence, est la première essence feuillue française; les 8 espèces indigènes (Robur, Petraea, Pubescens, Coccifera, Suber, llex, Pyrenaica, Cerris) rencontrées sur le territoire couvrent $41 \%$ de la surface boisée de production, soit 5,5 millions d'hectares. Le chêne pédonculé (Quercus robur $L$ ) avec ses 2,4 millions d'hectares et le chêne sessile (Quercus petraea Liebl) avec 1,8 million d'hectares, occupent une place privilégiée qu'ils doivent à leurs écologies respectives ainsi qu'à la diversité de leurs utilisations : du bois de feu à usage domestique ou industriel au bois d'œuvre destiné aux constructions civiles, au commerce intérieur (en particulier la tonnellerie), à l'ameublement ou... à la marine.

Ces usages variés dont le $X X^{\mathrm{e}}$ siècle retient essentiellement celui de production de bois d'œuvre de première qualité et en particulier de bois de tranchage, les prérogatives des différents propriétaires forestiers (forêts royales, seigneuriales, communales, ecclésiastiques, privées), les impératifs économiques du moment et enfin la volonté depuis le XIXe siècle d'imposer le mode de gestion de la futaie régulère ont contribué à façonner nos peuplements de chêne.

Qu'en est-il précisément de ce paysage en 1992 ? Comment a-t-il évolué depuis le XIVe siècle ? Comment doit-il évoluer?

\section{LA RESSOURCE EN CHÊNE ROUVRE ET PÉDONCULEE D'APRĖS LES DONNÉES DE L'INVENTAIRE FORESTIER NATIONAL (IFN) *}

Par la suite, et parce qu'elle constitue une source d'imprécision supplémentaire, la distinction entre chêne pédonculé et chêne

* Source : cellule d'évaluation de la ressource de l'IFN. rouvre ne sera pas faite par rapport aux définitions d'espèces prépondérantes ou pures ces 2 espèces seront confondues.

Seuls sont retenus les peuplements à chêne pédonculé et rouvre prépondérants (couvert relatif le plus grand sur le point d'inventaire).

\section{Quels en sont les propriétaires?}

Soixante-treize pourcent de la surface des chênaies se trouvent dans le domaine privé, contre $27 \%$ dans les forêts soumises; la répartition par propriétaire des chênaies est ainsi tout à fait représentative de ce qu'on observe pour la forêt française, toutes essences confondues.

\section{QUELS MODES DE GESTION LEUR APPLIQUE-T-ON?}

Les données de I'IFN sont une précieuse source d'information qui trouvent ici une première difficulté d'interprétation. Le sylviculteur, pour caractériser la gestion d'un peuplement, parlera de traitement en futaie régulière ou irrégulière, en taillis sous futaie ou en taillis, alors que les inventaires rendent compte uniquement et le mieux possible de l'état présent du peuplement, sans chercher à savoir quel mode de traitement le propriétaire a voulu appliquer. C'est ainsi en particulier qu'à la notion de traitement en taillis sous futaie, I'IFN substitue celle de mélange des 2 sous-unités taillis et futaie.

De plus, 2 types de démarche sont réalisées afin d'inventorier les peuplements; elles aboutissent d'une part à la définition de structures forestières de peuplement (évaluées sur le terrain sur une surface de 20 ares à 1 hectare), d'autre part à la définition de types de peuplement évalués par photo-interprétation. 
Dans le cas des chênaies, et étant donné les surfaces importantes qu'elles couvrent, il paraissait intéressant de confronter les résultats obtenus par ces 2 approches.

Quelques structures forestières et types de peuplement définis par l'IFN dont l'importance relative en surface était faible, ont été regroupés; les éléments retenus sont alors les suivants :

- en ce qui concerne les structures forestières : la futaie régulière pure (couvert relatif excède $75 \%$ ) ; la futaie régulière mélangée; le mélange taillis-futaie; le taillis et la coupe rase; la futaie irrégulière ;

- en ce qui concerne les types de peuplements: la futaie purement feuillue ; le mélange futaie à feuillus prépondérants et taillis; les taillis simples et assimilés; les boisements morcelés et assimilés (bois de ferme, parcs ruraux, champs boisés, boisements en timbre-poste); les autres types de peuplement: boisements lâches et assimilés, jeunes reboisements, futaie mixte, mélange taillis futaie à résineux prépondérant, futaie purement résineuse, qui mis ensemble couvrent des surfaces non négligeables ont été regroupés dans une catégorie "autres types".

Les principales divergences de résultats entre ces 2 inventaires concernent l'importance de la futaie régulière (fig 1) et son appartenance (fig 2). Admettre que la futaie couvre $9,79 \%$ (type de peuplement) ou $22,17 \%$ (structure forestière) des surfaces et qu'elles se situent en majorité dans les forêts soumises à $68,46 \%$ (type de peuplement) ou privées à $64,65 \%$ (structures forestières) sont des nuances de taille qui méritent quelques explications.

La différence entre ces 2 résultats a 2 origines.

La première, qui pourrait être considérée comme révélatrice d'un profond désarroi dans la gestion à suivre pour les chênaies, tient dans la divergence
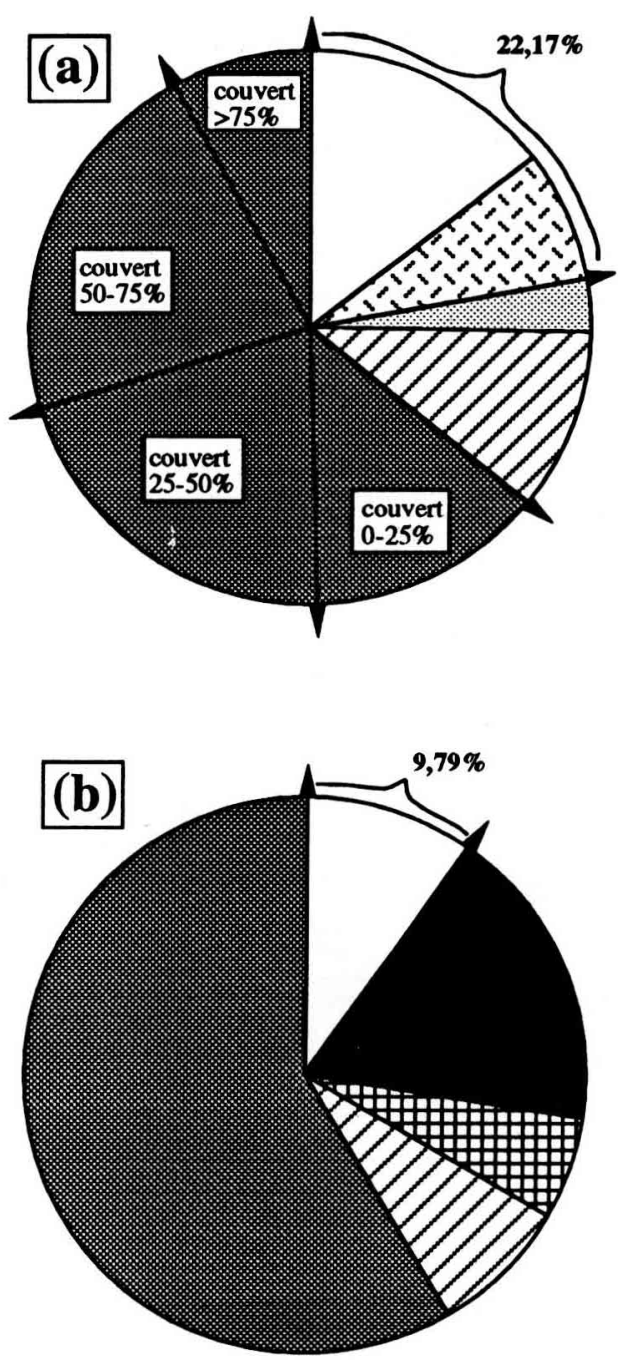

Fig 1. Répartition de la surface des chênaies de production (chênes rouvre et pédonculé prépondérants). a. Selon la structure forestière (les inventaires par structure permettent d'ordonner le mélange taillis-futaie selon la classe de couvert des réserves); $\square$ Futaie régulière pure; futaie régulière mélangée; futaie irrégulière; $Z$ taillis et coupe rase; mélange taillis-futaie. b. Selon le type de peuplement. $\square$ Futaie feuillue; boisement morcelé et assimilé; $\square$ autres types; $\square$ taillis simple et assimilé; mélange taillis-futaie à feuillus prépondérants. 
d'appréciation d'un même peuplement selon qu'elle résulte d'une photointerprétation (type de peuplement) ou d'une étude de terrain (structure forestière), et ce particulièrement dans le domaine privé (fig $3 \mathrm{a}, 3 \mathrm{~b}$ ). On trouve ainsi sur des suriaces importantes des bouquets régularisés dans le mélange taillisfutaie.

wrface (hectare)

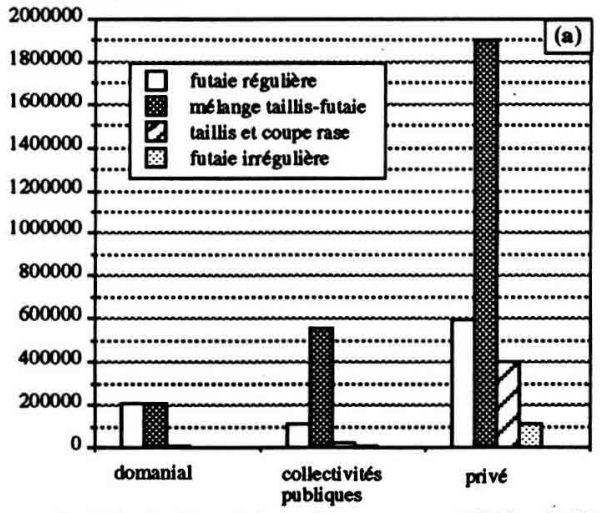

La futaie régulière prive represente avec cette definition $64,65 \%$ du total des futaies régutières.

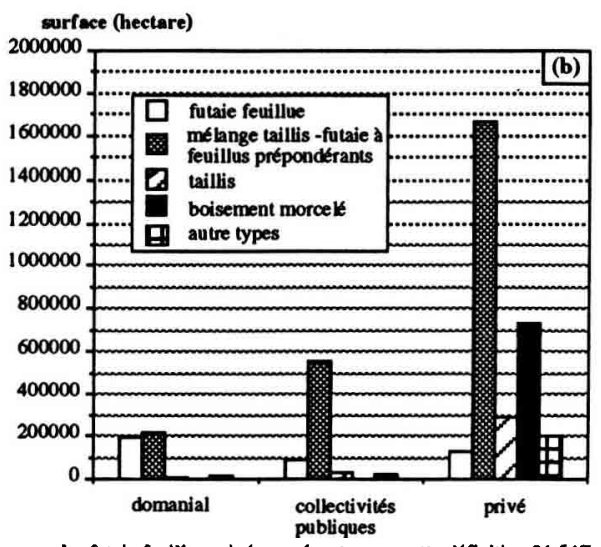

La futaie feuillue prive represente avec celte definition $31,54 \%$ du total des futaies.

Fig 2. Importance des principales structures forestières (a) et des principaux types de peuplement (b) selon le propriétaire.
La deuxième et la plus lourde de conséquence sur les résultats renseigne sur la structure de la propriété; I'IFN recense ainsi près de 735000 ha de boisements

(\%)

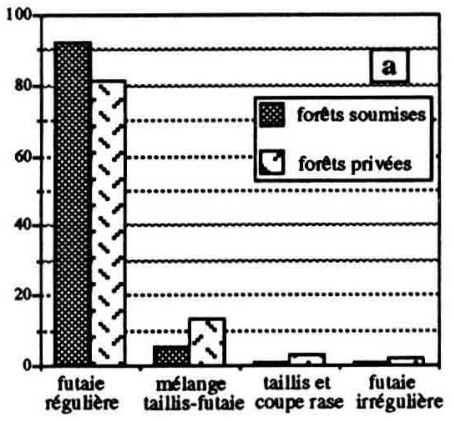

(\%)

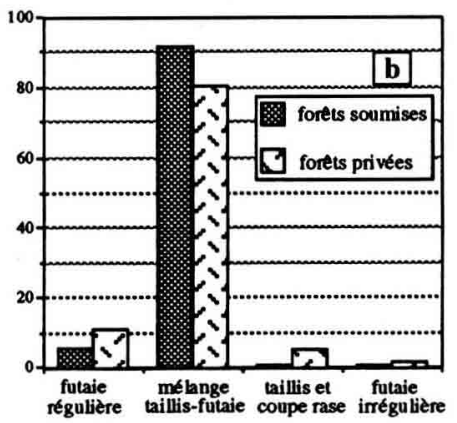

(\%)

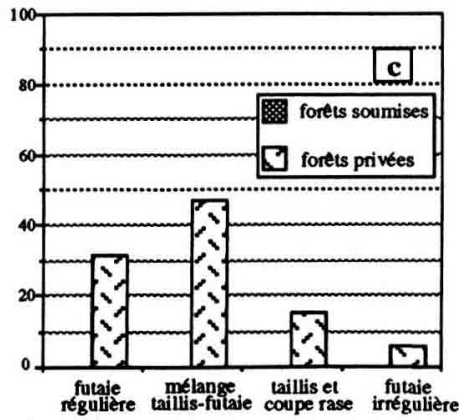

Fig 3. Correspondance entre type de peuplement et structure forestière pour les forêts "soumises" (domaniales et collectivités publiques) et les forêts privées. Répartition (en pourcentage) des surfaces de futaie feuillue (a), de mélange taillis-futaie à feuillus prépondérants (b) et de boisement morcelé et assimilé (c) dans chacune des principales structures forestières. 
morcelés (type de peuplement) pratiquement exclusivement dans le domaine privé (fig 2b, 3c), de même les "autres types" de peuplement n'appartiennent pratiquement qu'au domaine privé. Bien entendu, cela porte à réfléchir sur des modes de gestion appropriés à ces peuplements.

Le mélange taillis-futaie est le peuplement le plus important de nos chênaies puisqu'il représente en surface $58,73 \%$ (type de peuplement) ou $64,18 \%$ (structure forestière) (fig 1), l'essentiel se trouvant dans le privé (fig 4). Il est assez riche, principalement dans les forêts domaniales et celles des collectivités publiques, puisque dans près de la moitié des surfaces $(46,3 \%)$ le couvert des réserves dépasse $50 \%$ (fig 4).

II faut insister sur le fait que si le classement d'un peuplement par I'IFN en "mélange taillis-futaie" n'implique pas que la gestion en taillis sous futaie y soit appliquée actuellement, il en est cependant une émanation plus ou moins proche; la preuve la plus évidente en est les quelque 200000 ha de mélange taillis-futaie recen-

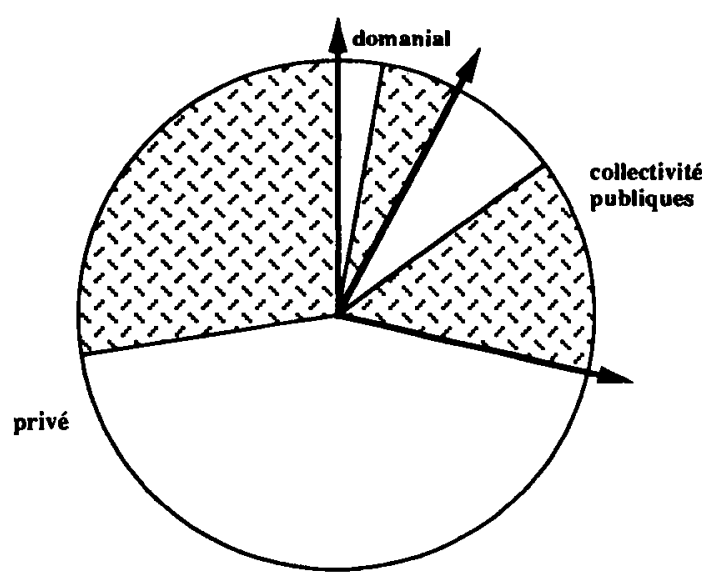

Fig 4. Répartition de la structure élémentaire : mélange taillis-futaie ( 2 classes de couvert représentées) par type de propriétaire. $\square$ Couvert des réserves inférieur à $50 \%$; $C$ Couvert des résenves supérieur à $50 \%$. sés par l'IFN en forêt domaniale, dont on sait par ailleurs que les premières étapes de la conversion ont été décidées.

Les 2 approches "type de peuplement" et «structure forestière" sont complémentaires; cependant notre préférence va à l'approche "type de peuplement" dans la mesure où, en distinguant les boisements épars, elle offre une image plus précise des peuplements susceptibles d'avoir une gestion suivie.

Comment en est-on arrivé à cette omniprésence du taillis sous futaie tant décrié depuis des siècles et dont la croyance forestière commune veut que Colbert ait porté un premier coup en privilégiant la futaie régulière?

\section{LE PASSÉ DE LA SYLVICULTURE DU CHÊNE EN FRANCE}

II ne fait pas de doute que le chêne, qu'il soit rouvre ou pédonculé, a bénéficié, très tôt dans notre pays, d'une faveur et d'un prestige très supérieurs à toutes nos autres essences forestières. Son importance actuelle, en forêt et hors forêt, est due à sa grande plasticité et à la volonté des hommes. En effet, les qualités mécaniques et la durabilité de son bois en ont fait une essence aux innombrables usages, sans oublier que pendant des siècles cet arbre "à fruits» a largement contribué, par le panage des porcs, à la survie des hommes.

Rien d'étonnant donc que nos ancêtres aient très tôt cherché à se l'approprier puis à le préserver et enfin à le cultiver. Nous allons tenter d'évoquer l'histoire de sa sylviculture telle que les textes anciens et l'observation de nos quelques rares peuplements multiséculaires nous permettent de la décripter. Il va sans dire que nous nous bornerons à une évocation historique 
très succincte, largement inspirée par l'étude historique des méthodes de l'aménagement forestier en France d'Huffel (1926).

\section{Du premier code forestier (1376) au début de la révolution industrielle}

Du XIVe au XVIII siècle, plus précisément jusqu'aux écrits de naturalistes et de forestiers éminents tels que Duhamel du Montceau, Buffon, Varenne de Fenille pour ne citer que les plus connus, la sylviculture ne constituait pas comme aujourd'hui un corps de doctrines. Les concepts techniques étaient noyés dans une réglementation policière à laquelle ils paraissaient très subordonnés. Les instructions régissant ce que nous appelons aujourd'hui l'aménagement des forêts étaient constituées d'une part par des réglements particuliers propres à chaque forêt et d'autre part par des ordonnances royales prises périodiquement après une sévère remise en ordre des services forestiers: les fameuses et redoutées "réformations".

Ces instructions ne préconisaient pas de techniques sylvicoles particulières. Elles se contentaient d'ordonner ou de réglementer (d'où leur nom) la récolte des bois, taillis ou grumes, dans le cadre très restreint des modes d'exploitation consacrés par l'usage dans chaque province. En plaine, où le chêne constituait l'essence noble, le mode d'exploitation en vigueur depuis la fin des grands défrichements (correspondant au début de la pénurie de bois en France) et entériné par l'ordonnance de Melun de 1376 était celui du "tire et aire". Ce système d'exploitation par assiette juxtaposée s'est peu à peu imposé, au détriment du furetage, pour des raisons d'efficacité dans le contrôle des coupes et de simplicité dans l'évaluation de la possibilité. Le furetage consistait à choisir les arbres à exploiter selon les be- soins et sans règles précises ; ce qui, dès que la demande se faisait pressante, conduisait aux plus grands abus. La coupe par assiette avait un autre avantage : elle rendait possible la mise en place d'une protection (clôture) des renaissances, semis ou rejets, contre la dent du bétail qui pullulait dans les forêts de l'époque.

En pratique, le tire et aire ressemblait fort à nos aménagements en taillis sous futaie et en taillis simple, selon que l'on réservait ou non des arbres épars lors du passage en coupe. Ces arbres réservés avaient un double rôle: produire du bois d'œuvre et assurer la part de la régénération constituée par un semis naturel de graines.

Les règlements d'exploitation se contentaient de préciser : 1) la révolution, c'est-àdire la périodicité de la coupe rase du taillis (les ordonnances ont imposé un minimum de 10 ans). II faut noter que le choix de la révolution conditionnait automatiquement la surface de l'assiette annuelle de la coupe; 2) le cas échéant, le nombre d'arbres à réserver (les ordonnances ont imposé un minimum de 16 à 20 baliveaux par hectare d'essence "chênes" prioritairement).

Après la répression des délits, le problème majeur que devaient résoudre les forestiers de l'époque était celui de la production de gros bois qui faisaient alors cruellement défaut à notre économie. En effet, la réserve d'arbres épars au moment de la coupe était rarement respectée dans les faits. Les réformations successives, jusqu'au XVIIe siècle, malgré la rigueur des peines infligées aux contrevenants ainsi qu'aux personnels forestiers convaincus de prévarication, n'ont pas réussi à faire de ces "arbres sur taillis" une source suffisante de bois d'œuvre.

If fallut donc trouver une autre solution. Plutôt que de procéder à la réserve d'arbres isolés jugés trop vulnérables, on décida de mettre en réserve tout un canton 
de forêt pendant un temps suffisamment long pour l'obtention de gros diamètres. Toute coupe intermédiaire était bannie par crainte de retomber dans les abus que l'on voulait justement éviter. Les ordonnances précisaient que ce canton devait être choisi sur les meilleurs stations et couvrir, depuis l'ordonnance de 1573, le quart de la surface de la forêt. Enfin, la durée de "mise en défends" devait être comprise, selon la fertilité du terrain, entre 100 et 200 ans.

Une autre solution, tout à fait équivalente, consistait simplement à allonger les révolutions du tire et aire. II semble toutefois que cette solution ait rarement été appliquée dans les faits tant les besoins immédiats en bois énergie étaient incontournables. Au XVIIIe siècle par exemple des révolutions initiales de 100 ou 150 ans ont été abaissées à 60 , voire 40 ans !

En conséquence on peut considérer que les "quarts en réserve", auxquels il faut ajouter les cantons de forêt réservés exclusivement aux chasses royales, ont constitué nos premières futaies de chêne. Certes, il ne s'agissait pas de vraies futaies régulières puisque les cantons réservés comportaient au départ des arbres d'âges très inégaux auxquels allait s'ajouter un recru naturel de semis et rejets, complété parfois par des regarnis artificiels.

Nous possédons encore quelques reliques de ces vieux peuplements nés du vieillissement d'anciens cantons traités initialement à tire et aire. C'est le cas de la réserve Colbert en forêt domaniale de Tronçais, dont l'origine est attestée par l'âge variable des arbres (250 à 350 ans) et par des traces d'élagage tardif qui trahissent sur certains d'entre eux une origine "taillis sous futaie". Citons également, parce qu'elles sont bien connues, les réserves artistiques de Réno-Valdieu dans l'Orne et des Beaux-Monts en forêt de
Compiègne. L'ensemble de ces peuplements, qui comptent parmi les plus vieux que nous possédons en France, ne couvrent actuellement guère plus d'une centaine d'hectares.

II serait toutefois erronné de croire que nos anciens se contentaient de simples coupes périodiques de récolte et ne connaissaient pas l'intérêt des opérations sylvicoles éducatives. Des recépages, forme primitive de nos dégagements de semis, sont pratiqués dès le XVIe siècle au moins. Des coupes "d'éclaircissement" ou "d'expurgade" sanitaires et sélectives sont parfois préconisées dans les règlements particuliers, mais toujours bannies par les ordonnances royales. Curieusement, Duhamel du Montceau, après en avoir fait l'éloge, donne raison aux ordonnances de les avoir interdites par crainte des abus; en effet, aux XVle et XVIIe siècle il était courant, car financièrement intéressant pour le personnel forestier, que soient confondues les coupes d'amélioration (recépages et expurgades) des renaissances de futaie avec de simples coupes réglées de taillis. Quant aux repeuplements artificiels, s'ils étaient peu pratiqués car très vulnérables dans le contexte de forêts pâturées, ils n'étaient pas inconnus et même préconisés par les ordonnances pour repeupler les cantons dégarnis.

II a fallu toutefois attendre le XVIIIe siècle pour que soient entrepris à grande échelle des reboisements en chêne. Les semis et plantations réalisés sur plusieurs milliers d'hectares dans les forêts de Compiègne et de Fontainebleau, pour ne citer que les principales, à la fin du XVIIIe siècle, ont constitué sans doute nos premières vraies futaies régulières de chêne d'une certaine ampleur. Ces futaies ont actuellement entre 200 et 250 ans et sont le plus souvent de haute qualité.

On ne peut, lors de cette évocation historique de nos chênaies, passer sous silence l'œuvre de Colbert. Contrairement à une opinion fort répandue, l'ordonnance de 
1669 n'apporte aucune innovation sylvicole particulière et se contente de reprendre, certes sous une forme améliorée et clarifiée, l'essentiel des dispositions contenues dans les ordonnances antérieures. L'apposition du quart en réserve par exemple était déjà prescrite au XVIe siècle.

If est vrai en revanche que la reconstruction de la flotte a constitué le prétexte à la mise en cuvre de cette grande réformation. Qu'on en juge par les volumes de chênes nécessaires à l'entretien de la marine militaire, soit environ $80000 \mathrm{~m}^{3}$ annuels (il s'agit des besoins de la marine sous Louis XVI, très proches de ceux de l'époque de Louis XIV) relativement aux énormes besoins du commerce intérieur. Ainsi, la seule tonnellerie consommait au milieu du XIXe siècle $400000 \mathrm{~m}^{3}$ (Broilliard, 1871) ; Nanquette (1868) déclarait même : "C'est que cette fabrication (du merrain)... suffirait à elle seule pour absorber une grande partie des chênes que la France produit".

On a dit à juste titre que les futaies pleines issues des mises en réserves prônées par Colbert n'ont pas produit le bois de marine espéré, mais plutôt du bois à texture faible, donc à résistance mécanique et durabilité médiocres. Colbert, pas plus que ses collaborateurs, n'ignorait quelle qualité de bois réclamaient ses arsenaux. Son objectif allait sans doute bien au-delà de la reconstruction de la flotte. La forêt française était dans un tel état de délabrement qu'il fallait frapper fort et imposer des mesures draconniennes pour sa remise en état, et, comme nous l'avons dit plus haut, le seul moyen efficace pour $y$ parvenir et notamment pour produire du bois d'œuvre était la mise en réserve quasi intégrale de certains cantons.

Dans le préambule à l'ordonnance de 1669, Louis XIV écrivait à propos des forêts : "Le ciel a tellement favorisé l'application de 8 années [depuis la fermeture des forêts décidée en 1661 par Colbert] que nous avons donné au rétablissement de cette noble et précieuse partie de notre domaine que nous le voyons aujourd'hui en état de refleurir plus que jamais et de produire avec abondance au public tous les avantages qu'il peut en espérer, soit pour les commodités de la vie privée, soit pour les nécessités de la guerre, soit enfin pour l'ornement de la paix" ; n'imaginons pas pour autant que les mesures prises par Colbert aient pu mettre un terme rapide à notre dépendance en matière première venant de l'étranger.

Ainsi, les constructeurs de bateaux à Copenhague (Danemark), Glückstadt (Allemagne), Göteborg (Suède) continuèrent à travailler pour Louis XIV. Durant les années 1662 à 1668, 119 vaisseaux furent achetés par la France, la plupart d'entre eux construits à l'étranger (les danois vendirent même des vaisseaux rendus inutiles par la conclusion de la paix dans le Nord) (Bamford, 1956). II est probable que c'est plutôt Louis XVI qui a profité des mesures conservatoires de bois de gros diamètre. $\mathrm{Ne}$ cachons pas non plus que l'ordonnance de 1669 était «à juste titre prévoyante quand elle autorisait l'approvisionnement de la marine dans les bois autres que les forêts royales et l'administration de la marine forte de cette ordonnance ne se laissait pas arrêter par les réclamations dont les procédures remplissent les archives forestières des abbayes, des communes et des particuliers". Telle était la conclusion de Picard (1875), qui dans l'analyse des comptes du trésorier de Bourgogne relatifs à l'approvisionnement de l'arsenal de Toulon, mettait en évidence sur la période de 1715 à 1723 que sur 17 384 arbres livrés, $57 \%$ provenaient des forêts particulières, $31 \%$ des forêts écclésiastiques, $10 \%$ des forêts communales, et seulement $2 \%$ des forêts royales.

Contre toute attente ce ne sont pas les futaies pleines qui ont le plus bénéficié des 
mesures prises par l'ordonnance de 1669. En effet, au XVIIIe siècle les besoins énormes en bois de feu de l'industrie naissante (forges, verreries, salines...) ont imposé une réduction brutale des révolutions des futaies qui sont devenues, de ce fait, de simples taillis sous futaie. En revanche, grâce à un personnel forestier très supérieur en moralité et capacité à celui qu'avait connu Colbert lors de sa prise de fonction, le recrutement des 20 "baliveaux" par hectare a été respecté. En Bourgogne par exemple, comme l'a si bien analysé l'historienne Andrée Corvol (1984), les réserves de taillis sous futaie se sont multipliées pour devenir aussi denses que des «bataillons de grenadiers". Ce qui a permis à Louis XVI de construire, avec des bois français, la plus belle flotte de l'époque.

\section{La conversion des taillis et taillis sous futaie}

Avec le XIXe siècle va progressivement s'estomper, dans notre pays, cette énorme pression de l'homme sur la forêt qui jusqu'alors avait le plus souvent réduit à néant les sages décisions prises par les ordonnances royales. La reconstitution patiente de nos forêts en général et des forêts de chêne en particulier va pouvoir s'amorcer.

Dès le début du XIXe siècle un consensus s'établit entre les forestiers et le pouvoir politique en faveur de la conversion des taillis sous futaie en futaie pleine.

La première technique utilisée, dès 1826 en Forêt de Senonches, consistait très simplement, après une brève période d'attente, à régénérer le peuplement de taillis sous futaie par le système des coupes progressives. Ce système de régénération naturelle qui n'était pas inconnu en France avait été clairement exposé par les forestiers allemands Hartig (1795) et
Cotta (1836) dans leurs traités de sylviculture parus au début du siècle. Son promoteur français B Lorentz eut le tort de vouloir aller trop vite en supprimant dès le départ toute coupe de taillis sous futaie sur l'ensemble de la forêt à convertir. La réduction brutale des revenus et des ventes de bois de feu qui en résultait provoqua la réaction du lobby des maîtres de forges qui réussirent à convaincre l'administration forestière, sinon d'arrêter tout au moins de réduire considérablement les mises en conversion.

Au milieu du XIXe siècle, une seconde méthode fut utilisée. Elle consistait en une coupe rase suivie d'un repeuplement artificiel. Cette méthode de "conversion directe" ne nécessitait pas de période d'attente et maintenait les coupes de taillis sous futaie sur le reste de la forêt. Le coût des travaux et les nombreux échecs enregistrés conduisirent à son abandon vers 1880 .

C'est à Parade que revient l'honneur de proposer en 1860 dans la quatrième édition de son "Cours de culture des bois" une méthode techniquement et économiquement satisfaisante. Sa méthode, version simplifiée de celle de Cotta, était fondée sur le réensemencement naturel des peuplements de taillis sous futaie vieillis et l'amélioration par éclaircies périodiques des jeunes peuplements de futaie. Le surplus de la forêt continuait à être traité en taillis sous futaie, ce qui permettait de maintenir les revenus et la production de bois de feu à un niveau satisfaisant.

Malgré l'utilisation industrielle de la houille et du coke, le prix du bois de chauffage restait élevé si bien que l'intérêt économique des futaies pleines était toujours contesté. L'échec de certaines régénérations provoqua vers 1880 une seconde opposition à la conversion, opposition qui ne s'acheva vraiment qu'avec la première guerre mondiale.

Au cours du XIXe siècle, d'importants progrès techniques avaient été réalisés. 
On s'aperçut par exemple que pour entamer avec succès le renouvellement naturel, il fallait observer une période de préparation des peuplements suffisamment longue pour tripler le volume initial du taillis sous futaie. C'est la raison pour laquelle dès 1870 on remplaça progressivement les coupes temporaires de taillis sous futaie par des éclaircies préparatoires, dans un triple but:

- améliorer le peuplement par sélection des tiges ;

- récolter progressivement les vieilles réserves ;

- épuiser le taillis.

Les résultats obtenus après 140 à 160 ans de vieillissement ont été le plus souvent remarquables. En effet, comme l'ont confirmé des études récentes (Triou et Vacherat, 1989), la production de gros bois, lors de cette période de vieillissement des taillis sous futaie, s'avérait alors équivalente à celle des futaies vraies exploitées à 180 ans.

Les jeunes futaies faisaient l'objet de dégagements puis d'éclaircies périodiques. Ces éclaircies de type mixte restaient modérées dans leur intensité. On leur reproche aujourd'hui de n'avoir pas été suffisamment violentes; à la lumière des différentes expériences d'éclaircie installées depuis 1925 par la station de recherches et d'expériences forestières dans les principaux massifs de chêne de qualité, il apparaît qu'il était sans doute difficile de faire mieux.

Jusque vers le milieu $d u X X X^{e}$ siècle, les conversions n'ont concerné, à de rares exceptions près, que les forêts domaniales. Les régénérations n'ont vraiment débuté qu'après 1870, si bien qu'aujourd'hui (1992) la grande majorité de nos vraies futaies de chêne ont leurs peuplements les plus vieux âgés de moins de 120 ans (fig 5) et ne couvrent, comparativement aux anciens taillis sous futaie, qu'une très faible surface. L'IFN ne mesurant ou n'estimant, de manière systématique, l'âge des peuplements réguliers que dans le cas des structures forestières, on notera que la répartition des surfaces par classes d'âge de la figure 5 est connue pour la totalité des 920118 ha de futaie régulière (représentant $22,17 \%$ des surfaces des chênaies de production selon le classement en structure forestière) et seulement pour une fraction, certes importante : 89\%, des 406232 ha des futaies feuillues (représentant $9,79 \%$ des surfaces des chênaies de production selon le classement en type de peuplement).

Les seules méthodes d'aménagement utilisées en France aussi bien pour les forêts à convertir que pour les vraies futaies de chênes ont été des méthodes dites "par contenance". C'est-à-dire que depuis le tire et aire et jusqu'à nos actuelles méthodes du groupe strict et du groupe élargi, la possibilité, par contenance puis par volume, découle du seul choix de l'âge d'exploitabilité ou, hypothèse non moins fragile, de la durée de survie des peuplements.

La France possède plusieurs millions d'ha de forêts où le chêne (rouvre et pé-

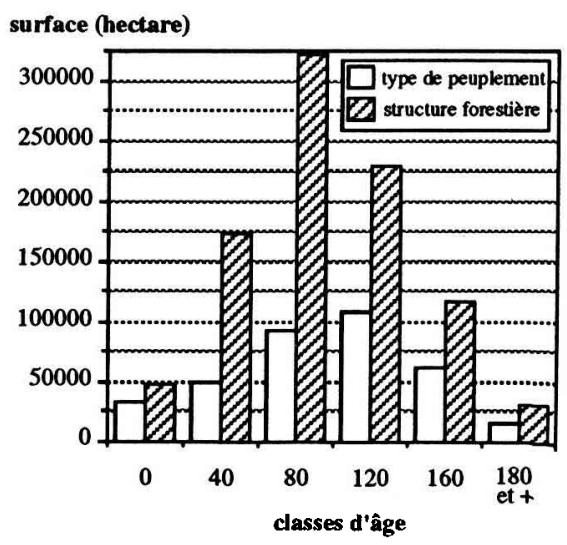

Fig 5. Répartition par classes d'âge des surfaces de futaie régulière (structure forestière) et de futaie feuillue (type de peuplement) de chêne. 
donculé) est l'essence prépondérante et qui représentent une richesse considérable tant au plan financier qu'au plan biologique et environnemental. Ces forêts ne sont pas des futaies régulières et ne sont pas domaniales. Pourquoi n'ont-elles pas été converties ? Et faut-il les convertir en futaie régulière?

\section{QUALITÉ DE LA RESSOURCE EN CHÊNE, VALEUR DE NOS FUTAIES ET DU MÉLANGE TAILLIS-FUTAIE}

Les données de l'IFN permettent d'apprécier la qualité des chênes présents dans les différents peuplements selon leurs dimensions (diamètre). Plusieurs catégories d'utilisation sont recensées: Q1 correspond au bois d'œuvre de qualité supérieure (tranchage, déroulage, ébénisterie, menuiserie fine), Q2 au bois d'œuvre de qualité inférieure (menuiserie courante, charpente, caisserie, coffrage, traverses) et enfin Q3 au bois d'industrie, de chauffage. Cet examen de la ressource présente l'avantage de l'objectivité puisqu'il ne sera pas question d'une sur- ou sous-exploitation de tel ou tel type de peuplement.

II ne s'agit pas de comparer strictement la futaie avec le taillis sous futaie (même si nous nous en étions défendu précédemment il faut quand même bien avouer que c'est à ce deuxième mode de gestion que l'on pense lorsqu'il est question de mélange taillis-futaie) ; en effet, nos données ne nous y autorisent pas. II faudrait comparer des stations identiques, ne retenir que les peuplements dont la gestion est représentative puis tenir compte en particulier des différences de vitesse de croissance, des frais de gestion, de la régularité des revenus, de l'impact paysager, autant d'éléments décisifs dans le choix d'un mode de gestion.

Non, il s'agit plutôt d'établir, avec les “imperfections" du classement de l'IFN, la valeur de la grume de taillis sous futaie par comparaison avec son homologue en futaie, cette dernière servant d'optimum de production supposé pour la qualité.

Les 2 approches, par structure forestière et par type de peuplement, ont été étudiées; elles conduisent en ce qui concerne la qualité des grumes à des résultats sensiblement identiques, seule l'approche par type de peuplement sera développée ici.
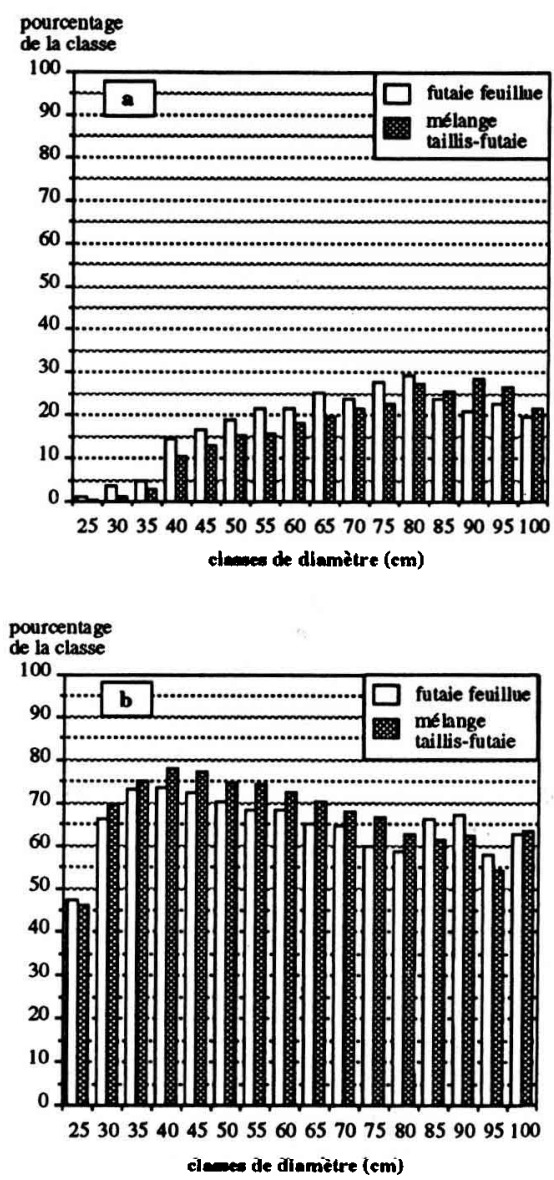

Fig 6. Estimation du volume de bois sur pied de qualité Q1 (a) et Q2 (b) par classes de diamètre (en pourcentage du volume total de la classe) pour la futaie feuillue et le mélange taillis-futaie à feuillus prépondérants. 
La comparaison des parts respectives des différentes qualités des produits Q1, Q2 et Q3 conduit à différencier difficilement la futaie feuillue du mélange taillisfutaie tous propriétaires confondus, avec un léger avantage pour la qualité Q1 en futaie (aucune pour la qualité Q2) (fig 6). Si on limite la comparaison à la seule forêt domaniale, dont la gestion est mieux suivie, on remarque que la proportion (en volume) de bois de qualité "Q1", tous diamètres confondus est de $14,8 \%$ pour la futaie et de $13,8 \%$ pour le mélange taillisfutaie.

Nos 2,4 à 2,7 millions d'ha de taillis sous futaie à base de chêne sont donc de valeur!

Les exploitants forestiers et les gestionnaires privés et publics le savent bien, eux qui quotidiennement sont en contact avec la réalité du terrain ou du marché !

Ne faut-il pas voir dans la valeur reconnue par les données de I'IFN des grumes de taillis sous futaie une explication satisfaisante aux conclusions surprenantes de l'étude de Demarcq (1981) sur les futaies à chêne de tranchage. II constatait en effet que sur le marché de la tranche (d'ailleurs très stable en volume) soit $85000 \mathrm{~m}^{3}$ consommés annuellement, à peine $20000 \mathrm{~m}^{3}$ provenaient des chênaies domaniales réputées (futaie et taillis sous futaie vieilli ou en conversion).

\section{DISCUSSION ET CONCLUSION}

Les données de I'IFN prenant en compte l'aspect extérieur de la grume et en particulier la présence et la dimension des nœuds (cachés ou non) dans le classement de la qualité, cela nous a conduit à nous interroger sur la confiance à accorder à la seule largeur du cerne comme prédicteur incontournable de cette qualité, à la croissance rapide des arbres de taillis sous futaie étant associée généralement une moindre qualité.

Un tour d'horizon de nos principales connaissances dans ce domaine paraît bien utile.

Tout d'abord, n'oublions pas que la relation entre densités du bois auxquelles sont liées les principales caractéristiques mécaniques du chêne et largeur du cerne sont des notions anciennes, bien connue, en particulier de nos constructeurs de navire. II est vrai qu'à l'époque, un mauvais choix de qualité avait souvent des conséquences désastreuses. Combien de fois, dans nos guerre maritimes, les conceptions militaires les meilleures n'ont-elles pas été déjouées par des avaries dans les coques des vaisseaux construits avec du mauvais bois (Coutance, 1870).

Ces constructeurs distinguaient déjà les bois gras à cerne étroit propres aux bordages car de faible rétractabilité, pièces faciles à changer et peu exposées à la pourriture, et les bois maigres à cerne large à forte rétractabilité, nerveux, destinés à la membrure du vaisseau. Bien entendu dans leur sagesse venaient se placer de nombreuses variétés à caractéristiques intermédiaires entre les bois nerveux et les bois gras. Malheureusement ils étaient avares d'indications sur les largeurs de cerne recouvrant ces notions de bois nerveux (forte densité) et gras (faible densité); la seule que nous ayons pu trouver provient du cours d'exploitation des bois de Nanquette (1868) : "Les bois de chêne nerveux (...) ont ordinairement les couches annuelles très développées et variant d'épaisseur entre $5 \mathrm{~mm}$ et $15 \mathrm{~mm}$ et au-dessus... sous ce rapport le chëne pédonculé paraît avoir l'avantage sur le chêne rouvre de France". Cette limite considérablement élevée explique peut-être qu'il n'y ait pas eu nécessité d'en faire un état chiffré, il suffisait de préciser qu'il était le plus souvent le fait d'arbres isolés (ou de tiges d'élite du taillis 
sous futaie) ayant poussé sur des terrains fertiles bien exposés. Comme Burger (1864), sous-inspecteur des forêts, le souligne avec justesse, "Le chêne de marine est un arbre de forme toute particulière (...) on pourrait bien se demander (à propos de sa culture) si c'est bien l'affaire du forestier, si ce n'est pas plutôt l'office de l'arboriculteur' (il pensait essentiellement aux bois courbes et courbants, nécessaires en quantité limitée, mais élément vital car constituant la structure du vaisseau).

Les bois, impropres au tranchage, car trop nerveux ne seraient-ils pas, en forêt, somme toute des arbres d'exception!

Les expériences réalisées depuis (Polge, 1973) ont confirmé pour ce qui concerne la densité du bois, à la fois cette extraordinaire variabilité individuelle pour des arbres dont les cernes annuels étaient en moyenne inférieurs à $4 \mathrm{~mm}$, ainsi que les remarquables qualités mécaniques des cernes étroits de largeurs moyennes comprises entre $0,5 \mathrm{~mm}$ et $1,5 \mathrm{~mm}$ constituant (l'étude étant réalisée sur carottes de sondage) les $15 \mathrm{~cm}$ en périphérie d'arbres âgés de 300 ans.

Polge (1973) constatait également une dégradation de la qualité (évaluée par l'augmentation de la densité du bois) pour un même arbre lorsqu'une série de cernes larges succédait à une série de cernes étroits. II concluait de cette étude que l'essentiel de la dégradation de la qualité se produisait lorsqu'on passait de cernes de $1 \mathrm{~mm}$ à $2 \mathrm{~mm}$. De même, une alternance de cernes larges et étroits était néfaste à la qualité. En conclusion, il fallait se garder de réaliser des éclaircies brutales et si la station le permettait, rester en-dessous de $2 \mathrm{~mm}$; au-delà et jusqu'à $5 \mathrm{~mm}$, la dégradation de la qualité restait plus limitée.

Quelles conséquences sylvicoles pratiques doit-on tirer de ces informations ? II s'agit n'oublions pas de tendances générales et je citerais Polge (1984) : “La dé- gradation de la qualité du bois avec la largeur de cerne ne présente pas de caractère systématique surtout lorsqu'on compare entre eux des individus d'une même parcelle"; on trouve encore et toujours cette extraordinaire variabilité individuelle...

Ainsi, Janin et al (1992), dans une étude sur les qualités esthétiques du chêne de tranchage, ont pu récolter sans difficulté chez un trancheur des échantillons présentant des largeurs moyennes de cerne allant de 1 à $7 \mathrm{~mm}$.

Dans ces conditions, faut-il se satisfaire du "faible» cerne moyen de l'ordre de 1,5 à $1,7 \mathrm{~mm}$, des chênes constituant le peuplement final de nos futaies régulières, dont on sait que la largeur est peu ou pas influencée par des éclaircies même fortes et assez précoces (Oswald, 1981 ; Ningre, 1990, 1993 à paraître), lesquelles éclaircies n'arrivant pas à régler la concurrence entre une multitude d'arbres dominants présentent par contre le défaut de créer épisodiquement des discontinuités de largeur de cerne; sachant également que même soumis à un régime d'éclaircie faible (donc peu influencé par le sylviculteur) on observe fréquemment pour les 100 plus gros chênes à l'hectare en futaie régulière des variations de cernes larges puis étroits dans la gamme de 1 à $2 \mathrm{~mm}$.

Ne faudrait-il pas, tout au contraire, valoriser au maximum nos stations forestières, dont le rôle (faut-il le rappeler !) dans la croissance du chêne est capital (Lanier, 1986) par le choix du traitement sylvicole le plus adapté : qu'il s'agisse de la futaie régulière, de la futaie jardinée ou du taillis sous futaie sans plus se soucier de la largeur du cerne comme l'admettent aujourd'hui bon nombre de nos voisins européens.

Et comme l'admettent en France les transformateurs du bois, rappelons les normes définies par l'APECF (Association 
pour la promotion des emplois du chêne français, 1989) fixant les largeurs de cerne admissibles, dans le commerce du chêne, pour le grain fin ou mi-fin :

"Un bois de grain fin a des cernes d'accroissement réguliers de valeur moyenne inférieure à $3 \mathrm{~mm}$ ".

"Un bois de grain mi-fin a des cernes d'accroissement réguliers pouvant aller jusqu'à 4,5 mm".

Pourquoi les sylviculteurs seraient-ils plus exigeants que les "utilisateurs" dans le domaine de la qualité.

Finalement, et en pratique, le premier critère de valeur d'un chêne "sans défaut" pour le marteleur comme pour le scieur qui ont en commun d'ignorer la largeur du cerne, ne serait-il pas son diamètre?

\section{RÉFÉRENCES}

APECF (1989) Guide pratique d'emploi des sciages de chêne et de hêtre français. Association pour la promotion des emplois du chêne français, Paris

Bamford PW (1956) Forests and French sea power 1660-1789. University of Toronto Press, Toronto

Broilliard C (1871) De la réserve des chênes d'avenir. Rev Deux-Mondes (15 sept)

Burger A (1864) Principes de culture et production régulière du chêne de marine. Rev Maritime Coloniale (août)

Coutance A (1873) Histoire du chêne dans l'antiquité et dans la nature. Baillière et fils, Paris

Corvol A (1984) L'homme et l'arbre sous l'ancien régime. Economica, Paris

Cotta $H$ (1836) Traité de culture forestière, $5^{e}$ édition. Roret-Heitz-Grimblot, Paris
Demarcq $P$ (1981) Les futaies à chêne de tranchage. Thème personnel d'étude, ENGREF, Nancy

Hartig G (1795) Anweisung zur Taxation der Forste, oder zur Bestimmung des Holzertrags der Wälder. Giessen, Darmstadt

Huffel $\mathrm{G}$ (1926) Les méthodes de l'aménagement forestier en France. Berger-Levrault, Paris

Janin G, Ningre F, Ihmoff A (1992) Les aspects des débits de chêne guidant le choix des professionnels du bois: résultats d'enquête. Colloque organisé par l'Association pour la promotion et la valorisation des activités du bois en Bourgogne (APROVALBOIS)

Lanier L (1986) Précis de sylviculture. École nationale du génie rural, des eaux et des forêts, Nancy

Nanquette $H(1868)$ Exploitation, débit et estimation des bois, $2^{e}$ edition. $V$ Raybois, Nancy

Ningre F (1990) Comparaison de différentes modalités d'éclaircie du chêne sessile. Premiers résultats d'un dispositif expérimental situé en forêt domaniale de Réno-Valdieu (Orne). Rev For Fr 2, 254-264

Oswald H (1981) Résultats des places d'expérience de chêne du Centre national de recherches forestières, $n^{\circ}$ spécial "Sylviculture en futaies feuillues". Rev For Fr 38, 65-85

Parade A (1860) Cours élémentaire de culture des bois, Paris Huzard, Nancy Grimblot, $4^{e}$ édition

Picard E (1875) L'approvisionnement de la marine sous la Régence 1715-1723. Rev Eaux Forêts 14, 171-176

Polge H, Keller R (1973) Qualité du bois et largeur d'accroissements en forêt du Tronçais. Ann Sci For 30 (2), 91-125

Polge $H$ (1984) Production de chênes de qualité en France, $n^{\circ}$ spécial «Dialogue forestier par dessus le Rhin". Rev For Fr 36, 34-48

Triou A, Vacherat E (1989) Approche économique de la conversion en région Centre. Rev For Fr 5, 431-438 